\title{
TO FIND THE PREVALENCE OF AUTONOMIC NEUROPATHY IN PATIENTS WITH NON ALCOHOLIC HEPATIC CIRRHOSIS
}

Deepak Garg, Devinder Singh Mahajan, Ashok Khurana, Jang Bahadur Singh

1. Junior Resident. Department of Medicine, Sri Guru Ram Das Institute of Medical Sciences and Research, Amritsar.

2. Professor. Department of Medicine, Sri Guru Ram Das Institute of Medical Sciences and Research, Amritsar.

3. Professor. Department of Medicine, Sri Guru Ram Das Institute of Medical Sciences and Research, Amritsar.

4. Senior Resident. Department of Medicine, Sri Guru Ram Das Institute of Medical Sciences and Research, Amritsar.

\section{CORRESPONDING AUTHOR}

\section{Dr. Deepak Garg,}

House no. 1843,

St. No. 8-G, Dasmesh Nagar,

Moga- 142001.

E-mail: deepaksir1985@yahoo.in

Ph: 00919646009050

ABSTARCT: Autonomic neuropathy has been reported in patients with alcoholic liver disease but information on its occurrence in patients with non-alcoholic liver disease is contradictory. To make the things more clear the present study was conducted to assess autonomic functions in patients with non-alcoholic liver disease and its relationships with the severity of liver damage. Autonomic function using five standard tests was examined in 50 cirrhotics. The extent of autonomic dysfunction was determined in the patients and a comparison between the characteristics of patients with and without autonomic neuropathy was made. Out of 50 patients $35(70 \%)$ were found to have autonomic neuropathy The alteration of the parasympathetic function [20 out of 35(57\%)] was significantly more frequent than that of sympathetic function [15(43\%)]. The prevalence of autonomic neuropathy was more $(80 \%)$ in patients with Child-Pugh grade $\mathrm{C}$ cirrhosis as compared to those having Child-Pugh grade $\mathrm{B}$ cirrhosis (54\%). The results were clinically significant but statistically insignificant ( $p>0.05)$. It was seen that out of total 50 cases of non alcoholic cirrhosis, the majority (68\%) were due to hepatitis C infection. A high prevalence of abnormalities in both sympathetic and parasympathetic function tests, and a poor relationship with liver function parameters, has been found in patients with non-alcoholic chronic liver disease.

KEY WORDS : Autonomic neuropathy, hepatic cirrhosis, hepatitis, Child Pugh class

INTRODUCTION: Cirrhosis leads to a number of complications, some of which eventually prove fatal. Autonomic neuropathy has been discovered to be one of such culprit. It is a known complication of many diseases such as diabetes and alcohol abuse. It has been well established in cirrhosis by various studies. Autonomic dysfunction in advanced liver disease is associated with decreased baroreceptor sensitivity to hypotension, leading to impaired blood pressure \& heart rate responses. 
MATERIAL AND METHODS: The study was based on 50 patients with non alcoholic hepatic cirrhosis.

\section{EXCLUSION CRITERIA:}

1. Subjects known to have consumed alcohol.

2. Subjects on drugs known to cause autonomic neuropathy like antihypertensives, anticholinergics, nitrates, ACE inhibitors, antidepressants, tranquilizers, barbiturates.

3. Those with a history of diabetes mellitus.

4. Ischemic heart disease and other medical conditions which could confound the interpretation of autonomic function tests in relation to cirrhosis.

5. Those unable to tolerate with drawl of diuretics one week prior to tests.

6. Those who were unable to cooperate during the performance of autonomic function tests were excluded from the study.

All patients were subjected to a battery of 5 standard autonomic tests as detailed below:

Tests reflecting parasympathetic damage:

1) Heart rate response to valsalva maneuver.

2) Heart rate (R-R) variation during deep breathing.

3) Immediate heart rate response to standing.

Tests reflecting sympathetic damage:

4) Blood pressure response to standing.

5) Blood pressure response to sustained handgrip.

Interpretation of tests was based on the works of Ewing and Clarke

Child and Turcotte (later modified by Pugh and named Child and Pugh) criteria were used in study to quantify the liver damage in the patients.

\section{ETHICS}

While performing this study on human subjects, the procedures followed were in accordance with the ethical standards of the responsible committee on human experimentation (institutional or regional) and with the Helsinki Declaration of 1975 that was revised in 2000.

RESULTS: The present study included 50 patients of hepatic cirrhosis who did not consume alcohol. There were almost equal number of male (44\%) and female (56\%) patients with majority of the patients in age group of 50-75 yrs. The mean age was $57.20 \pm 4.38$ years. This was seen that the major cause of non alcoholic cirrhosis was Hepatitis $\mathrm{C}$ accounting for $68 \%$ of the cases. Out of 50 cases of cirrhosis there was no patient in the Child-Pugh class A. The majority of the patients (80\%) belonged to Child-Pugh class B. Out of them $54 \%$ had evidence of autonomic neuropathy. $20 \%$ of the patients belonged to class C. Out of those, $80 \%$ had evidence of neuropathy ( $\mathrm{p}>0.05)$. Out of 50 cases, majority $35(70 \%)$ of the patients had either borderline $14(28 \%)$ or normal $21(42 \%)$ valsalva ratio. The abnormal value $(<1.10)$ was present in $15(30 \%)$ of the patients. The mean valsalva ratio was $1.166 \pm 0.035$. The minimum value was 0.97 and the maximum one was 1.64. Out of 40 patients of Child-Pugh class B, $30(75 \%)$ had an normal valsalva ratio whereas $10(25 \%)$ had an abnormal ratio. Out of 10 
patients of Child-Pugh class C, 5(50\%) had an normal valsalva ratio and 5(50\%) had an abnormal ratio. The results thus obtained were not significant with a p value $>0.05$.

Heart rate variation during deep breathing was normal ( $>15$ beats/min) in $70 \%$ of the patients out of the total 50 patients. There was no patient in the borderline group. $30 \%$ of the patients had an abnormal $(<10$ beats/min) heart rate variation. The mean value was $15.80 \pm$ 1.74 beats/min. The minimum value was 5.67 and the maximum was 26 beats $/ \mathrm{min}$. Out of 40 patients of Child-Pugh class B, 29(72.5\%) had an normal H-R variation during deep breathing whereas $11(27.5 \%)$ had an abnormal response. Out of 10 patients of Child-Pugh class C, $6(60 \%)$ had an normal H-R variation during deep breathing and $4(40 \%)$ had an abnormal response. The results thus obtained were not significant ( $\mathrm{p}>0.05)$.

Out of 50 patients, $50 \%$ had an abnormal heart response to immediate standing. Only $2 \%$ were in the borderline group, and the rest $48 \%$ had normal response. The mean value was $1.07 \pm 0.06$. The highest value was 1.92 and the lowest was 0.90 . Out of 40 patients of ChildPugh class B, $19(47.5 \%)$ had an normal immediate heart response to standing whereas $21(52.5 \%)$ had an abnormal response. Out of 10 patients of Child-Pugh class C, $6(60 \%)$ had an normal immediate heart response to standing (30:15) and 4(40\%) had an abnormal response. The results thus obtained were not significant with a $\mathrm{p}$ value $>0.05$

None of the patient had abnormal blood pressure response to standing.

It was evident in our study that $30 \%$ of the total patients had an abnormal blood pressure response to sustained hand grip. Rest $70 \%$ had an normal response. The mean value was $15.73 \pm 1.54$. The minimum value was 6.67 and the maximum was 24 . . Out of 40 patients of Child-Pugh class B), 28(70\%) had an normal blood pressure response to sustained hand grip (increase in diastolic pressure in $\mathrm{mm} \mathrm{Hg}$ ) whereas $12(30 \%)$ had an abnormal response. Out of 10 patients of Child-Pugh class C), 7(70\%) had an normal B.P response to sustained hand grip (increase in diastolic pressure in $\mathrm{mm} \mathrm{Hg}$ ) and $3(30 \%)$ had an abnormal response. The results thus obtained were not significant with a $\mathrm{p}$ value $>0.05$.

DISCUSSION: In the present study, 35 out of 50 cases (70\%) of non alcoholic hepatic cirrhosis were found to have an abnormal result in one or more autonomic function tests. 10 cases $(20 \%)$ had evidence of early parasympathetic damage, 10 cases (20\%) had definite parasympathetic damage and 15 cases (35\%) had combined (i.e both sympathetic and parasympathetic damage). These observations are consistent with the studies done by Gentile et al, 3 Hendickse, Thuluvah and Triger et al.4 Out of the 10 patients with early parasympathetic damage, $6(60 \%)$ belonged to Child-Pugh class B whereas 4 (40\%) to Child-Pugh class C. While in 10 patients with definite parasympathetic damage, 9 (90\%) belonged to Child-Pugh class B whereas $1(10 \%)$ to ChildPugh class $\mathrm{C}$ and in 15 patients with combined damage, 12 (80\%) belonged to Child-Pugh class B whereas $3(20 \%)$ to Child-Pugh class C. In total of 35 patients having autonomic neuropathy, $27(77 \%)$ belonged to Child-Pugh class B, whereas $8(23 \%)$ belonged to Child-Pugh class C.

In the present study there was no patient in the Child-Pugh class $\mathrm{A} .80 \%$ of the patients belonged to Child-Pugh class B. $20 \%$ of the patients belonged to class C. Out of $80 \%$ patients of Child-Pugh class B, 54\% had evidence of neuropathy. Out of those $20 \%$ patients of Child-Pugh class $\mathrm{C}, 80 \%$ had evidence of neuropathy. The mean C-P score was $8.70 \pm 0.32$ ( $>0.05)$. These findings are similar to observations of Bajaj5 and Oliver6 studies which reported increasing frequency of autonomic dysfunction with increasing severity of liver damage.

In the present study, heart rate response to standing was the most frequently ( 25 out of 50 patients) abnormal tests in cirrhotics. This is in consistence with the study by Barter and 
Tanner7 in their study on 30 patients of chronic liver disease in relation to autonomic neuropathy noted the heart-rate response to standing as the most sensitive test with high specificity.

The results were clinically significant but most probably due to a significant variation in number of patients in Child Pugh class A, B and C ( p = 0.315) the results came out to be statistically non significant.

CONCLUSION: The burden of cirrhosis in India is enormous with majority of them presenting in later stages of the disease. Hepatitis $C$ has emerged to be a major cause of non alcoholic cirrhosis. Autonomic neuropathy is present in nearly 70 percent of the patients which is a potential cause of many of the complications. An exhaustive clinical examination and some cost effective screening tests are sufficient to detect this problem in earlier stages, which can be instrumental in better care of the patients.

\section{REFRENCES:}

1. Laennec RTH. Traite de l' auscultation mediate. Paris, Chaude, 1826:196

2. Rossle R. In: Henke F, Lubarsch O, eds. Handbuch der Spezillen Pathologischen Anatomie und Histologie, vol 5, part 1. Julius Springer, Berlin. 1930

3. Gentile S. Autonomic neuropathy in liver disease: relationship with alcoholic etiology and severity of disease. Italy J Gastroenterol 1994;(2):53.

4. Hendrickse MT, Thuluvath PJ, Triger DR. Natural history of autonomic neuropathy in chronic liver disease. Lancet 1992;339:1462

5. B K Bajaj, M P Agarwal, B Krishna Ram. Autonomic neuropathy in patients with hepatic cirrhosis. . Postgrad Med J 2003;79:408-411

6. Oliver, E.M Brunt, et al. Nonalcoholic steatohepatitis: histologic features and clinical correlations with 30 blinded biopsy specimens. Hum Pathol.2004;35(9):1070-1082

7. Barter F, Tanner AR. Autonomic Neuropathy in an alcoholic population. Postgrad Med J 1987;63:1033. 


\begin{tabular}{|c|c|c|c|}
\hline & \multicolumn{2}{|c|}{ Child-Pugh class } \\
\hline & & $\mathrm{B}$ & $\mathrm{C}$ \\
\hline \multirow{12}{*}{$\begin{array}{l}\text { Autonomic } \\
\text { Neuropathy }\end{array}$} & 0 No. of Patients & 13 & 2 \\
\hline & $\%$ of patients with no neuropathy & $86.7 \%$ & $13.3 \%$ \\
\hline & $\%$ of patients within respective Child-Pugh classes & $32.5 \%$ & $20.0 \%$ \\
\hline & 1 No. of Patients & 6 & 4 \\
\hline & $\%$ of patients with early parasympathetic damage & $60.0 \%$ & $40.0 \%$ \\
\hline & $\%$ of patients within respective Child-Pugh classes & $15.0 \%$ & $40.0 \%$ \\
\hline & 2 No. of Patients & 9 & 1 \\
\hline & $\%$ of patients with definite parasympathetic damage & $90.0 \%$ & $10.0 \%$ \\
\hline & $\%$ of patients within respective Child-Pugh classes & $22.5 \%$ & $10.0 \%$ \\
\hline & 3 No. of Patients & 12 & 3 \\
\hline & $\%$ of patients with combined damage & $80.0 \%$ & $20.0 \%$ \\
\hline & $\%$ of patients within respective Child-Pugh classes & $30.0 \%$ & $30.0 \%$ \\
\hline \multirow[t]{3}{*}{ Total } & No. of Patients & 40 & 10 \\
\hline & $\%$ within autonomic neuropathy & $80.0 \%$ & $20.0 \%$ \\
\hline & $\%$ of patients within respective Child-Pugh classes & $100.0 \%$ & $100.0 \%$ \\
\hline
\end{tabular}

(0-No neuropathy. 1- early parasympathetic damage,

2- definite parasympathetic damage, 3- combined damage) 